\title{
The accuracy of the Acuros XB algorithm in external beam radiotherapy - a comprehensive review
}

\author{
Jarkko Ojala ${ }^{1,2}$ \\ ${ }^{1}$ Department of Oncology, Tampere University Hospital (Tays), Tampere, Finland \\ ${ }^{2}$ Department of Medical Physics, Medical Imaging Center, Tampere University Hospital (Tays), Tampere, Finland
}

Received October 13, 2014; Revised October 22, 2014; Accepted October 22, 2014; Published Online October 24, 2014

\section{Review Article}

\begin{abstract}
The accuracy of dose calculation algorithms has been a topic of interest among the radiotherapy community throughout last decades. On one hand the advancements in computers and algorithms has improved the accuracy, but on the other hand the developments in other parts of treatment process, in treatment delivery techniques and in treatment devices have always pushed the requirements to the next level. In this review article a comprehensive overview on the accuracy of a new type ' $c$ ' dose calculation algorithm, the Acuros XB (AXB) algorithm (Varian Medical Systems, Inc., Palo Alto, CA, USA), is provided. All the articles that have applied the AXB algorithm in terms of external beam radiotherapy are included and the research frames with reported deviations to reference methods are described. For the homogeneous water phantoms the reported accuracy was from $1 \%$ to $2 \%$, being of similar level for heterogeneous phantoms, in rare occasions lower. In anthropometric and anthropomorphic phantoms the mean deviations were about $2 \%$ and slightly larger for single points and/or small regions. With patient plans the reported average discrepancies were less than from $3 \%$ to $5 \%$. Almost without exceptions, the algorithm has proven to perform better than other existing commercial dose calculation algorithms. The number of such papers, in which the AXB algorithm is the only dose determination method, is already notable, which indicates that the accuracy of the algorithm is trusted for reference use and it also, with reported dosimetric results, implies that the AXB algorithm has reached its maturity.
\end{abstract}

Keywords: Acuros XB; AXB; External Beam Radiotherapy; Dosimetry; Monte Carlo

\section{Introduction}

The significance of dose calculation accuracy in the radiotherapy delivery process has increased during past decades. The introduction of CT images in radiotherapy allowed the inclusion of detailed anatomical geometry and material information in dose calculations. However, clinical dose calculation algorithms relied for a long time to the measurements in water and simplified corrections for patient contour and heterogeneities. These so called correction-based algorithms, also known as type 'a' algorithms are nowadays mostly replaced by type ' $b$ ' algorithms that are model-based to a varying degree, using superposition and convolution techniques. Type 'b' algorithms have provided increased accuracy over type 'a' algorithms in majority of clinical situations, but in the extreme ends of the density range of biological tissues (e.g., air, lung and bone) and in high-Z implanted materials (here, high- $Z$ materials are regarded as materials with larger mass density than found in human tissues), they have been shown to produce clinically unacceptable discrepancies, when comparing to reference methods. In the Report 85 from Task Group 65 of American Association of Physicists in Medicine $(\mathrm{AAPM})^{1}$ the importance of the dose calculation accuracy was demonstrated by determining the proportion of each source of uncertainty in the whole radiotherapy treatment process. At that time (year 2004) the accuracy was estimated to range from $1.0 \%$ to $5.0 \%$, but to decrease below $3.0 \%$ in the future. However, while other sources of uncertainty were estimated to decrease as well, the relative contribution of the dose calculation accuracy was to increase. During past decade the developments in radiotherapy have taken place in treatment delivery, in the form of intensity modulated radiotherapy (IMRT) and volumetric modulated arc radiotherapy (VMAT), which use treatment fields applying small field segments generated by multi-leaf collimators (MLC), and in treatment protocols, which favor hypofractionation, i.e., large treatment doses per fraction delivered to small target volumes. These advancements have also posed a demand for more accurate dose calculation, which has been realized as a new generation of algorithms that are based on fast Monte Carlo (MC) methods or so called grid-based linear Boltzmann transport equation (LBTE) solvers. Characteristic for the most recent class of algorithms, type 'c', is that 1) the modelling of secondary electron transport has been developed to next 
level, when comparing to type 'a' and 'b' algorithms, 2) they are able to calculate the dose deposition, in addition to all biological tissues, also in the presence of high- $\mathrm{Z}$ implanted materials and 3) the dose is reported as dose to medium ${ }^{2,3}$.

The first addition to this group of algorithms and the only commercial grid-based LBTE solver for radiotherapy purposes is the Acuros XB (AXB) algorithm implemented in the Eclipse treatment planning system (TPS) by Varian Medical Systems, Inc. (VMS) (Palo Alto, CA, USA). ${ }^{4}$ Originally, the roots of the AXB algorithm lie in the Attila LBTE solver developed and benchmarked by a group based on the founders of the Transpire, Inc. (Gig Harbor, WA, USA).5, 6, 7, 8 The original Acuros LBTE solver designed for radiotherapy dose calculations was developed by Transpire, Inc. from the Attila ${ }^{9}$, which was then implemented as the AXB algorithm in the Eclipse TPS ${ }^{4}$. The AXB algorithm was developed to address the clinical needs of dose calculation accuracy comparable to full MC methods with moderate calculation times, especially with treatment plans with large number of field segments, such as in VMAT. The BTE is a coupled system of integrodifferential equations that describe the transport and interactions of various particles (photons, electrons, neutrons, etc.) in matter in macroscopic level. When expressed in linearized form it is assumed that the transport of each particle is independent of other transported particles and no electromagnetic fields are present. As analytical, closed form solutions for LBTE in calculation geometries typically applied in radiotherapy are not known, non-analytical, open form solutions must be applied. With grid-based LBTE solvers the solution is achieved with numerical methods, with which in ideal case the solution would only contain the error produced by the uncertainties in the particle interaction data. However, the core idea with the grid-based LBTE solvers is to discretize the variables in space, angle and energy, which generates systematic error and the total, combined uncertainty from abovementioned sources is defined by the compromise between the level of discretization and desired calculation speed. In addition, a small uncertainty is generated, since the AXB algorithm, similar to MC algorithms, employs energy cutoff values for photon and electron transport, which means that the particle transport is terminated and energy is absorbed locally, when the energy of a particle falls below the set cutoff values. ${ }^{4}$

The radiotherapy treatment plan dose calculation with the $\mathrm{AXB}$ algorithm can be divided in two phases. In the first phase the radiation beam propagation in the linear accelerator treatment head is simulated. The AXB algorithm uses the same sub-source models as implemented in the Analytic, Anisotropic Algorithm (AAA), i.e., the model contains sub sources for: 1) primary photons, which are generated in the $\mathrm{X}$-ray target, but not interacted elsewhere in the treatment head, 2) extra-focal photons, which are generated in interactions in other treatment head components, and 3) electron contamination, which represents the electrons generated in the treatment head components and in the air. ${ }^{10,11,12,13,14,15}$ The radiation beam fluence determined by the source model, modulated by the plan-specific jaw and MLC configurations, is then directed to the patient, which is the first step in the second phase of the dose calculation. In the patient dose calculation, the following steps are performed: 1) transport of source model fluence into the patient, 2) calculation of scattered photon fluence in the patient, 3) calculation of scattered electron fluence in the patient, and 4) dose calculation. Step 1 ) is repeated for each field direction and steps 2) to 3) are performed only once for each patient geometry voxel. In the final step 4) the absolute dose in each voxel is calculated using the determined electron angular fluence, macroscopic electron energy deposition cross sections and material density of the voxel. ${ }^{4,9}$ Since the AXB algorithm performs explicit simulation of physical interactions in matter, the material mapping needs to be done for the CT-based patient geometry prior to abovementioned steps. Mass density and material type are defined for each voxel in the material mapping, applying CT simulator specific CT number to mass density conversion curve and material library provided with the AXB algorithm. The material library includes five tissue types and 16 other materials, the mass density upper limit being $8.0 \mathrm{~g} / \mathrm{cc}$ for stainless steel. ${ }^{4}$ Thus, the report mode for the final dose distribution is referred to as dose-to-medium in medium $\left(D_{m, m}\right)$. Although the AXB algorithm inherently calculates $\mathrm{D}_{\mathrm{m}, \mathrm{m}}$, the dose distributions can be converted to dose-to-water in medium $\left(D_{\mathrm{w}, \mathrm{m}}\right)$, which is done by replacing the medium-based fluence-to-dose response function used in absorbed dose calculation with a water-based response function. In the type 'a' algorithm implemented in Eclipse TPS, the pencil beam convolution (PBC) algorithm and in the AAA, which is a type ' $b$ ' algorithm, the dose report mode is also $D_{w, m}$, but in those algorithms the dose results are based on electron density-based corrections applied to dose kernels calculated in water. ${ }^{4,}$, 16 Therefore $D_{\mathrm{w}, \mathrm{m}}$ mode of the AXB algorithm represents more closely true absorbed dose to water. ${ }^{17}$

A more rigorous and detailed description of the AXB algorithm is provided in the white paper from VMS by Failla et $a l^{4}$ and in the first papers benchmarking the accuracy of the original Acuros algorithm ${ }^{9}$ and the AXB algorithm ${ }^{17,} 18$. Kan et al. ${ }^{19}$ have provided a review article on grid-based LBTE solvers (i.e., the AXB algorithm), which presents the results of selected earlier papers describing the validation of the $\mathrm{AXB}$ algorithm for clinical use. The purpose of this review article is to present all the papers to date (Sep 2014) in which the AXB algorithm has been benchmarked, but also to cover such papers, where the dose distributions are solely produced by the AXB algorithm. To include all the articles implementing the AXB algorithm, the literature search was performed using PubMed and Scopus databases, but also a search was conducted at the website of each relevant journal, in order to include also those journals that are not included in the data- 
bases. Separate searches for keywords 'Acuros' and 'AXB' were performed in each case. The information on different dose calculation algorithms and measurements is included, as is the information on the research frame, e.g., what kinds of phantoms were used, what was the underlying clinical context of the work. The results between the AXB algorithm and the reported reference method (or assumed by the author of this article) are presented, but due to the limited space, for the results for other algorithms the reader is encouraged to consult the original paper. The presented AXB calculation results are in $\mathrm{D}_{\mathrm{m}, \mathrm{m}}$.

\section{The AXB algorithm and homogeneous phantoms}

The benchmarking in a homogeneous phantom is the first step in the validation process of a new dose calculation algorithm. Therefore, for new algorithms on the market, such as the AXB algorithm, it has been done in the most of the first publications that have included the algorithm. A pre-requisite for this is that the reference dose determination method, being a measurement and/or other dose calculation algorithm, has to be able to provide accurate, reference results with small uncertainty in the calculation geometry that is studied. Since all dose determination methods include an inherent uncertainty, the extent of this error needs to be known for accuracy assessment of the AXB algorithm.

The first validation of the AXB algorithm (versions 10 and 11) in homogeneous water phantom was presented by Fogliata et al. ${ }^{18}$ For 6 and 15 MV flattened beams and 6 and 10 MV flattening filter free (FFF) beams for field sizes (FSs) ranging from $2 \times 2$ to $40 \times 40 \mathrm{~cm}^{2}$ the reported uncertainties were within $1 \%$ for open beams and $2 \%$ for beams with mechanical wedges, when compared with measurements and the AAA. Full MC algorithms, such as EGSnrc/BEAMnrc/DOSXYZnrc, Geant, Penelope and MCNP, when appropriately commissioned, are considered to represent the most accurate means to determine dose distributions, especially in complex 3D phantoms including heterogeneities. ${ }^{20}$ In a study by Bush et al. ${ }^{17}$ the AXB algorithm version 10 (the AXB10) was benchmarked for 6 and $18 \mathrm{MV}$ beams against full MC simulations, measurements and the AAA. For FSs ranging from $4 \times 4$ to 30 $\times 30 \mathrm{~cm}^{2}$ in regions excluding large dose gradients the discrepancies were within $\pm 2 \%$, usually much less, between all the methods. Similar findings are presented for the AXB10 in paper by Han et al. ${ }^{16}$, where also type 'b' collapsed-cone convolution (CCC) algorithm implemented in Pinnacle 9.0 TPS (Philips Medical Systems, Cleveland, Inc., Fitchburg, WI, USA) was included. In a study by Hoffmann et al. ${ }^{21}$ prior to clinical implementation the AXB10 was benchmarked for 6 and $15 \mathrm{MV}$ beams against output factor (OF), percentage depth dose (PDD) and profile measurements and the corresponding AAA calculations. Results for output factors against measurements were within $1 \%, 1 \%$ and $1 \mathrm{~mm}$ distance to agreement (DTA) for PDDs and $2 \% / 1 \mathrm{~mm}$ in profiles excluding penumbrae. Mißlbeck and Kneschaurek ${ }^{22}$ compared the AXB10 to the AAA and XVMC fast MC algorithm implemented in iPlan TPS (BrainLab AG, Feldkirchen, Germany), qualitatively stating that there were no large differences in PDDs for $10 \times 10 \mathrm{~cm}^{2}$ field with 6 and $15 \mathrm{MV}$ beams.

The trend in recent past in radiotherapy has been to treat small tumors with conformal static MLC-shaped fields, applying hypofractionation, i.e., using high dose fractions instead of conventional fractionation schemes. At present, these so called stereotactic body radiotherapy (SBRT) and stereotactic radiosurgery (SRS) treatments are delivered with IMRT and VMAT techniques, which use even smaller MLC-shaped apertures than in treatments with static MLC-shaped fields. All this has increased the importance of the dose calculation accuracy in small field apertures, especially because with smaller number of treatment fractions the relative importance of each fraction increases correspondingly. To address this, there are several papers that concentrate on the accuracy of the AXB algorithm with small fields and following issues. Fogliata et al..$^{23}$ benchmarked the accuracy of the AXB11 and the AAA against measurements with static jaw-collimated $6 \mathrm{MV}$ fields ranging from $0.8 \times 0.8$ to $3 \times 3 \mathrm{~cm}^{2}$ and several VMAT fields with varying jaw-collimation. The accuracy of the AXB11 was within $0.2 \%$ and gamma agreement index (GAI) values higher than $95 \%$ were achieved for FSs larger than $1 \times 1 \mathrm{~cm}^{2}$. For smaller fields the discrepancies were larger. A white paper from VMS by Torsti et al. ${ }^{24}$ assessed the dose calculation accuracy of the AAA, the AXB10 and the AXB11 with small MLC-delimited fields, presenting also the influence of several parameters included in algorithm configuration and dose calculation options. With $2 \% / 1 \mathrm{~mm}$ GAI acceptance criteria the calculation results against measurements were good for all field configurations excluding the field with $1 \times 1 \mathrm{~cm}^{2}$ MLC-collimation and $2 \times 2 \mathrm{~cm}^{2}$ jaw-collimation. The effect of the air cavity correction option in Eclipse VMAT optimization engine was tested by Kan et al. ${ }^{25}$ and the final VMAT plan dose calculation was performed with the AXB10 and the AAA. In water phantom the AAA resulted in slightly higher dose coverage in planning target volume (PTV) and near-maximum dose in organ-at-risk (OAR).

Whenever there are MLC-delimited static or dynamic fields, there is dose contribution due to the jaw and MLC transmission and interleaf gap leakage radiation. Kron et al. ${ }^{26}$ and Fogliata et al. ${ }^{27}$ assessed the accuracy of the AXB11 and the AAA under the shielding by the jaw, the MLC or the both with varying static and VMAT fields. In general, good results were obtained for both algorithms, the largest discrepancies being for the smallest fields. In this context, the effect of the value of an $\mathrm{AXB}$ algorithm configuration parameter (also present in the AAA) called 'effective spot size', in addition to MLC parameters (transmission and dosimetric leaf gap), was highlighted and the tuning of the parameter is also discussed in Torsti et al. ${ }^{24}$. Additionally, there are two other studies, where the AXB algorithm has been used in conjunction with 
homogeneous water phantoms. In work by Tan et al..$^{28}$ the AXB10 was compared with the PBC algorithm and the AAA implemented in Eclipse TPS and FFT convolution algorithm and multigrid superposition algorithm implemented in $\mathrm{XiO}$ TPS (Elekta AB, Stockholm, Sweden) and VMC fast MC algorithm implemented in Monaco TPS (Elekta AB). The algorithms were used to determine the entrance and exit doses to be used in in vivo dosimetry. In comparison to point dose measurements, the $\mathrm{AXB10}, \mathrm{XiO}$ algorithms and Monaco produced deviations of $2-3 \%$, whereas the deviations for the PBC algorithm and the AAA were about $5 \%$. In profile and 2D comparison the VMC algorithm performed better than the AXB10. Jeevanandam et al. ${ }^{29}$ applied the AXB11 with the AAA for benchmarking the in-house independent monitor unit verification calculation for VMAT. The AXB11 was compared with total scatter factor and tissue maximum ratio measurements, with mean deviations of $0.83 \%$ and $-0.15 \%$, respectively. However, the further results in the study reveal that the improved accuracy of the AXB11 produces larger differences between the in-house dose calculation program and the AXB11 than compared to the AAA.

\section{The AXB algorithm and heterogeneous phantoms}

The second step in validation process of a new dose calculation algorithm is to assess the accuracy of the algorithm in heterogeneous phantoms, i.e., phantoms that contain different materials. The structure of the phantom may consist of slabs or layers of various materials, which are perpendicular to beam axis. In this case the accuracy of the heterogeneity correction of the dose calculation engine is tested mainly in forward direction. When including heterogeneities of different size and shapes in various locations in the phantom, the performance of the heterogeneity correction is also benchmarked in lateral directions. This way the phantom may also represent better anatomical geometries and by choosing tissue-equivalent materials to the phantom, the results are more effectively transferable to clinical setting.

In the report by Vassiliev et al. ${ }^{9}$ the predecessor of the AXB algorithm, Acuros algorithm by the Transpire Inc. was compared in heterogeneous phantom against full MC simulations for 6 and $18 \mathrm{MV}$ beams with FSs ranging from $2.5 \times 2.5 \mathrm{~cm}^{2}$ to $10 \times 10 \mathrm{~cm}^{2}$. The phantom, so called 'ICCR benchmark' ${ }^{30}$, consisted of slabs of water, bone and lung. The maximum differences were $1.2 \%$ and $2.3 \%$ for 6 and $18 \mathrm{MV}$ beams, respectively. For $18 \mathrm{MV}$ beam the largest difference was for the smallest FS - for all other fields the maximum difference was $1.1 \%$. In profiles the differences were of similar order and the DTA values were less than $1 \mathrm{~mm}$. In the white paper by Failla et al. ${ }^{4}$ the AXB algorithm was shown to have very good visual agreement with full MC simulations in slab phantoms containing various materials from the AXB algorithm material library. Bush et al. ${ }^{17}$ studied the performance of the AXB10 against full MC calculations and the AAA with 6 and $18 \mathrm{MV}$ beams and several FSs in unit-density phantoms con- taining air or lung with varying density and bone representing ribs. The deviations between the AXB10 and full MC simulations were within $\pm 2-3 \%$ for the phantoms with lung and $\pm 1.5-4.5 \%$ for the phantom with air, with only very small regions of larger discrepancies, usually occurring at the interface between the lung/air and tissue parallel to the beam. The results for the phantom containing lung were cross-checked against measurements with similar findings by Kroon et al. ${ }^{31}$ In the work by Han et al. ${ }^{16}$ the AXB10 was compared to the AAA, the CCC algorithm implemented in Pinnacle TPS and full MC simulations for 6 and 18 MV beams in multilayer slab phantom containing soft tissue, bone and lung. The average differences for the AXB10, when compared to the full MC simulations, were $1.1 \%$, which was better than with other algorithms. Similar results were achieved by Fogliata et al. ${ }^{32}$, who compared the AXB10 and the AXB11 against $\mathrm{VMC}++$ fast $\mathrm{MC}$ algorithm and the AAA in phantoms containing air, lung of varying density, adipose, skeletal muscle, cartilage and bone. With $3 \% / 3 \mathrm{~mm}$ acceptance criteria the $\mathrm{GAI}$ values in comparison to $\mathrm{VMC}++$ algorithm were $100 \%$ for lung with normal density and bone and $86 \%$ for light lung. In the paper by Mißlbeck and Kneschaurek ${ }^{22}$ the AXB10 was compared to the AAA and XVMC fast MC algorithm in iPlan TPS in water phantoms containing lung and bone. The calculated dose distributions showed only minor differences between the AXB10 and XVMC algorithm. The AXB11, the AAA and the XVMC algorithm were also benchmarked in a study by Tsuruta et al. ${ }^{33}$, accompanied with measurements. The deviations between the AXB11 and measurements were generally within $\pm 3 \%$, the maximum difference being $-5.5 \%$. Notable was that it was one of the rare studies, where other TPS algorithm, the XVMC in this case, achieved more accurate results than the AXB algorithm, when comparing to the reference method. Stathakis et al. ${ }^{34}$ assessed the accuracy of the AXB10 with small fields $(1 \times 1-5$ $\times 5 \mathrm{~cm}^{2}$ ) in water phantoms containing lung, bone or air inserts for $6 \mathrm{MV}$ beams by comparing to full MC simulations and the AAA and the CCC algorithm implemented in Pinnacle TPS. The agreement between the AXB10 and full MC simulations was within $2 \%$ for lung and bone, but for the phantom with air, the deviations were larger - ranging from 3 to $15 \%$. In study by Kan et al..$^{25}$, which tested the effect of the air cavity correction option in Eclipse VMAT optimization engine, calculations were also performed in heterogeneous phantoms. The improved accuracy of the AXB10 compared to the AAA resulted to that in the AXB10 dose distributions the PTV dose coverage was much lower and therefore the choice of the algorithm affects the subsequent results of the study. However, the final impact on patient treatment planning was not fully revealed by the study.

There are a couple of groups that have applied similar type of heterogeneous phantoms in several papers. In studies by Kan et $a .^{35,36,37}$, where the accuracy of the AXB10 for the nasopharyngeal carcinoma (NPC) treatment planning has been assessed, a rectangular phantom of variable size with variable 
size rectangular air cavity or bone insert in the middle and rod-type bone inserts on both sides of the air cavity has been used. The comparison has been done against measurements, full MC simulations and the AAA. The congruence of the AXB10 with the reference methods, where available, was generally good, maximum differences near air/tissue interfaces being $6 \%$ and the GAI value with $3 \% / 3 \mathrm{~mm}$ acceptance criteria being only once less than 96\%. Rana et al.38, 39, 40, 41 have benchmarked the accuracy of the AXB10 and the AAA with $6 \mathrm{MV}$ beams mainly for lung SBRT and radiotherapy of esophageal cancer in heterogeneous slab phantoms containing solid water and air gap, with additional lung insert in one study ${ }^{41}$. When comparing to measurements, the largest discrepancies for the AXB10 were $-3.9 \%$ near the air/water interface ${ }^{40}$, while the ranges for deviations were within $\pm 0.5 \%{ }^{39}$, from $-3.8 \%$ to $0.9 \%{ }^{38}$, from $-3.9 \%$ to $-0.4 \%{ }^{40}$ and from $-1.7 \%$ to $2.8 \%{ }^{41}$.

The accuracy of dose calculation algorithms in the presence of high-Z materials has been a challenge during past decades to the extent that it is general to avoid such beam directions from which the beam would primarily traverse through high-Z material, e.g., hip implants. Of course this is not always possible, e.g., in the case of dental implants, and alongside with the improvements in metal artifact removal (MAR) techniques, there is also a need for more accurate dose calculation. Some of the most advanced dose calculation algorithms to date are able to predict the beam attenuation in high-Z material to some extent, but none of them are able to model the backscatter peak in dose at the tissue/high-Z material interface. In the work by Failla et al. ${ }^{4}$ it was shown that the $\mathrm{AXB}$ algorithm is able to calculate the dose distribution in the presence of high- $\mathrm{Z}$ materials, such as aluminum, titanium alloy and stainless steel, when compared to full MC simulations. Lloyd and Ansbacher ${ }^{42}$ assessed the accuracy of the AXB11 against measurements, full MC simulations and the AAA in a cubic water phantom with stainless steel and titanium alloy inserts. There is a visual agreement with the measurements and both the full MC simulations and the AXB11. For the 6 and $18 \mathrm{MV}$ beams GAI values of $96.8 \%$ and 91.3\% were achieved between the full MC simulation and the AXB11, with acceptance criteria of $2 \% / 1 \mathrm{~mm}$.

\section{The AXB algorithm and anthropometric/anthropomorphic phantoms}

In the validation process of a new dose calculation algorithm the third step is the utilization of anthropometric phantoms, which mimic the anatomy of a patient, and anthropomorphic phantoms, which in addition to mimicking the anatomy, also make use of the tissue-equivalent materials. These phantoms represent the most complex types of phantoms, in which reference measurements may be performed (excluding the rare cases, where detectors are implanted in the patient, or when entrance or exit doses are measured on patient surface). Therefore, realistic patient treatment plans with measure- ment data in these phantoms are usually used for final benchmarking of new algorithms. However, the more complex the phantom, the more challenging it is to perform accurate measurements, especially in $2 \mathrm{D}$ or $3 \mathrm{D}$. Thus, the application of full MC simulations is a feasible option to comprehensive benchmarking.

In the fundamental study by Vassiliev et al. ${ }^{9}$ the accuracy of the Acuros algorithm was assessed by applying a breast treatment plan to an anthropomorphic phantom and comparing to full MC simulations. Using $2 \% / 2 \mathrm{~mm}$ as acceptance criteria, the GAI value was $98.7 \%$. Hoffman et al. ${ }^{21}$ compared the AXB10 to measured data from an anthropomorphic thorax phantom with a set of various treatment plans. For 6 and $15 \mathrm{MV}$ beams, with acceptance criteria of $3 \% / 3 \mathrm{~mm}$, the average GAI values were $98.2 \%$ and $99.5 \%$, respectively, all of them being over $95 \%$. Han et al. ${ }^{43}, 44$ have studied the accuracy of the AXB11 in head \& neck $(H \& N)$ and lung regions, using dedicated anthropomorphic phantoms. The comparison was made against measurements and the AAA for IMRT and VMAT treatment plans using $6 \mathrm{MV}$ beams. The agreement between the point dose measurements and the AXB11 were in ranges from $0.1 \%$ to $3.6 \%$ and from $0.4 \%$ to $4.4 \%$ for the $\mathrm{H} \& \mathrm{~N}$ and lung cases, respectively. GAI values of over $90 \%$ and $97 \%$ were achieved with acceptance criteria of $5 \% / 3 \mathrm{~mm}$ and $\pm 3 \% / 3 \mathrm{~mm}$ for the H\&N and lung cases, respectively. The same dedicated H\&N phantom was also used in the work by Failla et al. $^{4}$, where the deviations in point doses ranged from $0.5 \%$ to $4.6 \%$, averaging to $1.9 \%$. In studies by Kan et al. ${ }^{35}, 37$, where the accuracy of the AXB10 for the NPC treatment planning has been assessed, point and 2D dose distributions were compared to the AXB10 and the AAA in IMRT treatment plans applied to an anthropomorphic phantom. The reported discrepancies between the point dose measurements ranged from $0.7 \%$ to $2.6 \%{ }^{35}$ and from $0.0 \%$ to $5.5 \%{ }^{37}$, averaging to $1.4 \%$ and $1.7 \%$, respectively, while the GAI value was $91.3 \%$ with acceptance criteria of $\pm 3 \% / 3 \mathrm{~mm}^{37}$. In a work by Ojala et al. ${ }^{3}$ the accuracy of the AXB10 was benchmarked in an anthropometric pelvic phantom containing a titanium alloy implant. By applying a $6 \mathrm{MV}$ beam through the implant and comparing to measurements and full MC simulations in MAR-corrected CT dataset, the discrepancies between the AXB10 (in MAR-corrected CT dataset) and measurements ranged from $-5.5 \%$ to $1.8 \%$, averaging to $-2.0 \%$, while the deviations between the full MC simulations and measured data were from $-3.1 \%$ to $0.7 \%$, averaging to $-0.2 \%$. In addition, there are two studies, where the primary intention has not been to benchmark the AXB algorithm, but by including other calculation methods, a comparison between the methods can be established. Warren et al. ${ }^{45}$ studied the optimization of collimator margins in lung radiotherapy and used the AXB10 and the AAA for dose calculation. The improved accuracy of the AXB10 compared to the AAA resulted to that in the AXB10 dose distributions the PTV dose coverage was lower and therefore the effect of the choice of the algorithm on the subsequent results of the study needs to be assessed. In 
the work by Gimeno et $a .^{46}$ the AXB10 and the AAA were used to assess the accuracy of an electronic portal imaging device (EPID) -based in vivo volumetric dosimetry software, where the dose calculation was based on pencil beam principles. The improved accuracy of the AXB10 over the AAA and pencil beam algorithms revealed larger discrepancies between the AXB10 and the pencil beam algorithm than between the AAA and the pencil beam algorithm.

\section{The AXB algorithm and patient plans}

The fourth, final step in the validation process of a new dose calculation algorithm is to quantify how much the dose distributions of clinical patient plans change, when comparing to older TPS algorithms. Preferably there should be a comparison also to a reference method, which in the case of patient plans can only be another dose calculation algorithm, such as full MC simulations.

The largest number of studies in this category is related to the radiotherapy of the lung, mostly to lung SBRT. In the study by Bush et al. ${ }^{17}$ a single $18 \mathrm{MV}$ field was incident on the left lung and the AXB10 was compared to full MC simulations. The AXB10 passed the gamma test with $\pm 2 \% / 2 \mathrm{~mm}$ acceptance criteria and the $1.0 \%$ to $2.5 \%$ differences were partially related to different material assignments between the calculations. Tsuruta et al. ${ }^{33}$ benchmarked the AXB11 and the AAA with XVMC fast MC algorithm in iPlan TPS with lung SBRT treatment plans. The dose deviations between the AXB11 and the XVMC algorithm were less than 4\% for more than $94.6 \%$ of the points and in dose-volume histogram (DVH) parameters the differences were less than $2 \%$. Similar findings were reported by Tomiyama et al. ${ }^{47}$, who in addition to the AXB10, the AAA and XVMC fast MC algorithm in iPlan TPS, also included the PBC algorithm. The discrepancies between the AXB10 and the XVMC algorithm were within $3 \%$. Chetty et al. ${ }^{48}$ did not benchmark various dose calculation algorithms as such in their work, but studied the correlation of dose distributions with local control in stereotactic ablative radiotherapy (SABR). The included algorithms were the AXB11, the AAA, XVMC fast MC algorithm in iPlan TPS, two types of pencil beam algorithms and the CCC algorithm in Pinnacle TPS. The deviations in DVH parameter values between the AXB11 and the XVMC algorithm were less than $2.5 \%$. In the study by Stathakis et $a l^{34}$ jaw-collimated arc plans for lung and paraspinal lesion radiotherapy were used to benchmark the AXB10, the AAA and the CCC algorithm found in Oncentra MasterPlan against full MC simulations. With all the algorithms the deviations to full MC simulations ranged from $2 \%$ to $5 \%$, the AXB10 being one of the algorithms with smallest discrepancies. The only study, where the AXB algorithm has been benchmarked against full MC simulations in realistic lung SBRT treatment plans is by Ojala et al. ${ }^{2}$, where the AXB10, the AAA and the PBC algorithm were compared to each other, accompanied with a comparison of the AXB10 with full MC simulations. With
$3 \% / 3 \mathrm{~mm}$ acceptance criteria the 3D GAI values were over 95\% for all calculated treatment plans, but stricter criteria, DVH parameter and dose difference comparison revealed larger deviations, especially with small isolated target volumes.

Studies, where the AXB algorithm has only been compared to the AAA, usually through AXB-recalculated AAA treatment plans, are numerous. Fogliata et al..$^{49}$ assessed the clinical impact of the AXB11 in lung radiotherapy by comparing the dose distributions to the AAA. For the PTV the mean deviations were less than $2 \%$ and for the OAR less than $3 \%$. In the work by Kroon et al. ${ }^{31}$ the AXB10 was compared to the AAA in conventional lung radiotherapy and lung SBRT. The largest differences, up to $-12.3 \%$, in DVH parameter values were for the PTV minimum doses, while being less for other DVH parameters, averaging to the range from $-1.8 \%$ to $2.0 \%$. In two studies by Rana et al. ${ }^{40,41}$ the AXB10 was compared to the AAA in lung SBRT for varying-sized PTVs. To the PTV the AXB10 predicted lower mean and minimum doses $(0.3 \%$ and $4.3 \%$, respectively $\left.{ }^{40}\right)$ and higher mean maximum doses $(2.3 \%$ ${ }^{40}$ ), whereas higher doses to the ipsilateral lung and lower mean maximum doses to the spinal cord and to the heart. The more heterogeneous target dose distribution resulting with the AXB algorithm was also present in the work by Liu et $a .^{50}$, where the AXB11 was benchmarked in lung SBRT with the AAA. The dose heterogeneity also influenced to the amount of needed monitor units with the AXB11 to deliver the same prescription dose as with the AAA studied by Khan et $a l^{51}$ and to the collimator margin optimization studied by Warren et al. ${ }^{45}$.

Kan et al. have studied the dosimetric impact of the AXB algorithm in the radiotherapy of NPC. ${ }^{35,36,37}$ In the applied IMRT and VMAT plans originally calculated with the AAA and recalculated with the AXB10 common findings were that the AXB10 predicted lower target coverage, lower minimum target dose, lower mean target dose and also lower doses to OARs. In breast radiotherapy Fogliata et al. ${ }^{52}$ compared the AXB10 and AXB11 to the AAA in a typical two tangential 6 MV field setting. The findings were similar to NPC cases by Kan et al., i.e., the AXB algorithm predicted lower doses both to the PTV and to the lung. Interestingly, the paper by Fogliata et al. is the only study published that reports quantitatively the improved calculation accuracy of the AXB11 over the AXB10 in low density materials. Of two other studies related to breast radiotherapy in the first one by Lonski et al. ${ }^{53}$ the unknown version of the AXB algorithm was shown to be superior in out-of-field dose calculation, when comparing to measurements, the PBC algorithm and the AAA. In the other study by Petillion et al. ${ }^{54}$ the choice of dose calculation algorithm was shown to have an impact on the parameters of radiobiological models. They suggested that the most accurate solution would be to use the AXB algorithm in $\mathrm{D}_{\mathrm{m}, \mathrm{m}}$ dose report mode. 
There are also several reports available on assessing the accuracy of the AXB algorithm on various anatomical regions and with various clinical diagnoses. Rana et al. ${ }^{55}$ studied the clinical impact of the AXB10 over the AAA in prostate cancer VMAT treatments. In general, the AXB10 predicted lower dose, but the differences in DVH parameter comparison were within $1 \%$. In another paper by Rana et al. ${ }^{39}$ the AXB10 was benchmarked in the VMAT treatment of esophageal cancer. In DVH parameter comparison the AXB10 resulted in lower PTV coverage, lower mean and minimum PTV doses, but higher maximum PTV dose. Averaged mean doses to all OARs and maximum doses to heart, liver and spinal cord, calculated by the AXB10, were lower. The only DVH parameter value for OARs that was predicted to be higher by the AXB10 was the maximum lung dose. Fogliata et al. ${ }^{56}$ performed a dosimetric comparison between the AXB11, the AAA and proton radiotherapy for soft-tissue sarcoma. No clear differences were observed in the PTV dose distributions between the AXB11 and the AAA, though the number of needed monitor units by the AXB11 was $1.8 \%$ higher. Interestingly, the AAA calculation results were closer to the AXB11 $D_{m, m}$ calculations than $D_{w, m}$ calculations, even in the bone. In a work by Krayenbuehl et al. ${ }^{57}$ that studied the application of IMRT and VMAT for malignant pleural mesothelioma the role of the AXB algorithm was limited. Of all the patient cases only one was calculated with an unknown version of the AXB algorithm. The DVH parameter comparison to the AAA revealed deviations that all were within $1 \%$.

Couple reports include patient plans from multiple anatomical regions. Kathirvel et al. ${ }^{58}$ benchmarked the AXB10 and the AAA against measurement-based CCC calculations. The treatment plans for lung SBRT and head, H\&N, thoracic and pelvic regions were used for the study. For all the plans the average difference between the AXB10 and the CCC algorithm were less than $0.5 \%$ and GAI values of over $99.5 \%$ were achieved. In the study by Mißlbeck and Kneschaurek ${ }^{22}$, a liver, lung SBRT, and $H \& N$ patient cases were included to compare the AXB10 and the AAA to XVMC fast MC algorithm found in iPlan TPS. However, the qualitative analysis of the DVH data did not reveal such discrepancies between the algorithms that were anticipated from the results with the heterogeneous phantom. The first work to include the AXB13 was by Stroom et al.59, who studied the effect of isocenter positioning in SABR applying VMAT in the brain, lung and spine. Potentially clinically relevant discrepancies were found to be involved in the choice of dose calculation algorithm, the AXB11 being preferable. Finally, for the feature that in the AXB algorithm outperforms other commercially available algorithms, Lloyd and Ansbacher ${ }^{42}$ showed that the dose distribution in the presence of the high-Z hip implant is much closer to the full MC simulation with the AXB11 than with the AAA. In Ojala et al. ${ }^{3}$ the accuracy of the AXB10 was assessed by replacing the sector of a VMAT arc, from which the beam would traverse the hip implant and that is normally planned as an avoidance sector, by three static fields. The dose distribution was used as base dose plan in VMAT optimization to prepare a clinically acceptable plan. The plan was recalculated with full $\mathrm{MC}$ model and the AAA. The 3D GAI value between the AXB10 and the full MC simulations was $99.01 \%$ and the deviations in the dose distributions in the vicinity of the implant, but also elsewhere, were small, which can also be observed in Figure 1 (a)-(b). The differences in DVH parameters for the PTV were less than $1.5 \%$ and less than $4 \%$ in the OARs.

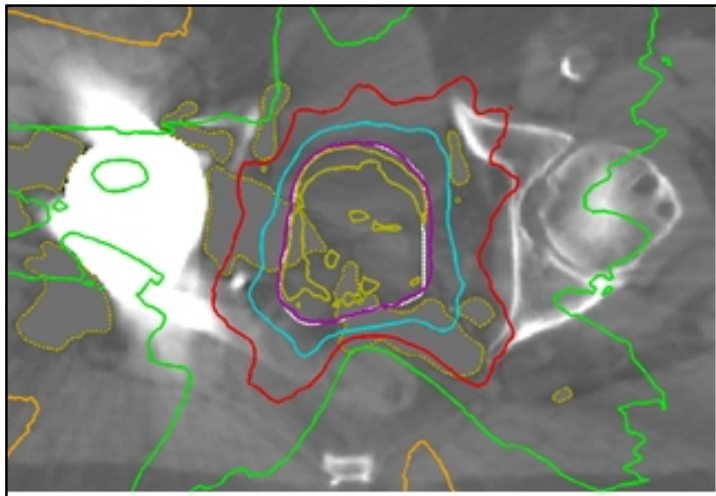

(a)

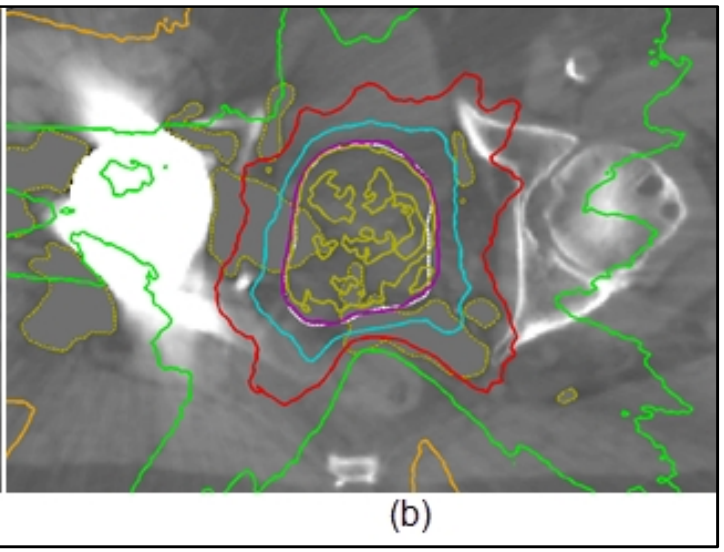

FIG. 1: The isodose curves for a clinical patient plan from Ojala et al. ${ }^{3}$, calculated both by the AXB algorithm (a) and the MC model (b). Isodose levels from outermost curve are 10\%,30\%, 50\%, 70\%, 95\% and 100\% of prescribed dose (PTV white). The constant grey areas represent where the HU correction is applied.

\section{The AXB algorithm as only dose determination method}

On top of all the previously presented studies, in which the intention has been to assess the accuracy of the AXB algorithm by comparing to reference methods and to reveal the discrepancies to other commercially available dose calculation algorithms, there is a number of reports, where the AXB algorithm has been the only dose determination method used. 
This implies that the authors have had faith in that the algorithm can be applied for reference use and to predict dose distributions, which are the basis for further research purposes.

Abacioglu et al. ${ }^{60}$ evaluated the role of VMAT technique for SRS of benign brain lesions in comparison to Gammaknife SRS. In the study the AXB10 was the only dose calculation algorithm for VMAT treatment plans. Fogliata et al. ${ }^{61}$ and Mancosu et $a .^{62}$ have studied the application of VMAT technique in total marrow irradiation and in the studies, the AXB10 was the only algorithm that was used. For the radiotherapy of the breast, another group has produced two papers, by Essers et $a l .{ }^{63}$ and Osman et $a .^{64}$, in which the application of VMAT and breath-hold techniques is assessed applying the AXB10. Apart from studies with clinical perspectives, Vanetti et al. ${ }^{65}$ studied the behavior of two versions of VMAT optimization algorithm implemented in Eclipse TPS and in the study, the AXB10 was used as the calculation algorithm for the final dose distributions. Persoon et al. ${ }^{66}$ applied an unknown version of the $\mathrm{AXB}$ algorithm treatment planning and predicting the delivered dose from portal dosimetry measurements. Finally, in study by Gersh et al..$^{67}$, the AXB11 was used to evaluate the impact of detector choice used in beam data measurements.

\section{Discussion}

Table 1 shows against which dose determination methods the AXB algorithm has been benchmarked and in what kind of situations. Studies for each column are needed in the validation process of a new dose calculation algorithm. Compari- sons to measurements and full MC simulations are needed, when the 'absolute' accuracy of the new algorithm is determined. To quantify the difference to existing commercial algorithms and following clinical impact, comprehensive benchmarking in various anatomical locations and clinical settings against other algorithms is required. Table 1 suggests that the AXB algorithm is comprehensively tested against the $\mathrm{AAA}$ and to some extent against measurements, but more comparisons against measurements would be beneficial especially for the AXB11 or the AXB13 in heterogeneous and more complex phantoms. Since the full MC simulations are recognized as the gold standard dose determination method, especially in geometries, where measurements are difficult or impossible to perform, more studies including full MC simulations are recommended. While the report on differences between the AXB algorithm and the AAA are numerous, more data on evaluating the clinical impact of the improved dose calculation performance in various clinical settings is needed. Even more beneficial for the radiotherapy community would be to include other advanced commercial dose calculation algorithms, such as fast MC implementations and the CCC algorithm, to comparisons.

There are no means to provide a simple quantitative description on the accuracy of a dose calculation algorithm. The reported 'accuracy' always depends on, e.g., what is the reference dose determination method and what is the related uncertainty, in what kind of phantom/setting the benchmark is performed and what is the metric used. Therefore it is always important to explore the original publication carefully until final conclusions are drawn.

TABLE 1: The distribution of the contents of various studies, including the information on how many times each version of the AXB algorithm is benchmarked with each type of phantom and against what dose determination method.

\begin{tabular}{|c|c|c|c|c|c|}
\hline & Homogenous & Heterogeneous & Anthropometric & Anthropomorphic & Patient \\
\hline \multirow[t]{2}{*}{ Meas. } & $\mathrm{XXXXXX}$ & $\operatorname{XxXXXXXX}$ & $\mathrm{x}$ & $\operatorname{xxxxx}$ & o \\
\hline & +++++++ & ++ & & ++ & \\
\hline \multirow[t]{2}{*}{ full-MC } & $\mathrm{xx}$ & $\operatorname{xxxXx}$ & $\mathrm{x}$ & - & $\mathrm{XXXX}$ \\
\hline & & +- & & & + \\
\hline fast-MC & $\mathrm{xx}$ & $\mathrm{xx}++$ & & & $\mathrm{xx}++$ \\
\hline $\mathrm{CCC}$ & $\mathrm{xx}$ & $\mathrm{xx}$ & & & $\mathrm{xx}+$ \\
\hline \multirow[t]{2}{*}{ AAA } & $\operatorname{XXXXXXXX}$ & 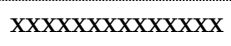 & & $\mathrm{XXXX}$ & 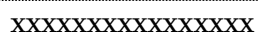 \\
\hline & ++++++ & +++ & & ++ & ++++++++ \\
\hline Type 'a' & $\mathrm{x}$ & & & $\mathrm{x}$ & $\mathrm{xx}++\mathrm{O}$ \\
\hline
\end{tabular}

Abbreviations: $\mathrm{x}=\mathrm{AXB} 10 ;+=\mathrm{AXB} 11 ; \#=\mathrm{AXB} 13 ; \mathrm{o}=\mathrm{AXB}$ ?; - = pre-AXB 
However, from all the covered studies several common quantitative observations were perceivable. In homogeneous water phantoms the reported deviations against measurements ranged from $1 \%$ to $2 \%$ and in areas of high dose gradient, e.g., penumbrae, DTA values of $1 \mathrm{~mm}$ were achieved. These results were for the FSs larger than $2 \times 2 \mathrm{~cm}^{2}$, whereas discrepancies were larger for smaller fields. For the heterogeneous phantoms the deviations in lung ranged commonly from $1 \%$ to $3 \%$, being slightly larger for light lung, and up $15 \%$ for air. Anyhow, the largest discrepancies occurred mainly for very limited regions and limited number of measurements points, usually near the interface. Also, in these regions the uncertainty of the reference method may be elevated and the largest reported deviations were for the AXB10, which has been shown to underperform in certain circumstances and which has been improved in the AXB11. With anthropometric and anthropomorphic phantoms the maximum dose differences against measurements were about $5 \%$, averaging to about $2 \%$ or less. For the patient treatment plan comparisons the relevance of the dose determination method played a larger role. In general, the reported average dose differences were up to $3 \%$ to $5 \%$, being of similar range for many DVH parameters and occasionally larger for minimum or maximum dose values, which represent point doses. Throughout the studies, reported GAI values were generally high, mostly over $95 \%$, depending on the selected acceptance criteria.

\section{Conclusions}

The number of studies, where the AXB algorithm has been used, is over 50. Almost without exceptions, the algorithm has proven to perform better than other existing commercial dose calculation algorithms. The number of such papers, in which the AXB algorithm is the only dose determination method, is already notable, which indicates that the accuracy of the algorithm is trusted for reference use. This, with reported dosimetric results, implies that the $\mathrm{AXB}$ algorithm has reached its maturity. It has been validated for intended use, with knowledge on the achievable accuracy. However, there are situations where the utilization of further verification methods, such as full MC simulations, is encouraged.

\section{Conflict of interest}

The author declares that he has no conflicts of interest. The author alone is responsible for the content and writing of the paper.

\section{References}

1. Papanikolau N, Battista JJ, Boyer AL, et al. Tissue inhomogeneity corrections for megavoltage photon beams. Report of Task Group No. 65 of the Radiation Therapy Committee of the American Associa- tion of Physicist in Medicine. AAPM Report No. 85. Available from: http://www.aapm.org/pubs/reports/rpt_85.pdf

2. Ojala JJ, Kapanen MK, Hyödynmaa SJ, et al. Performance of dose calculation algorithms from three generations in lung SBRT: comparison with full Monte Carlo-based dose distributions. J Appl Clin Med Phys 2014; 15:4662.

3. Ojala J, Kapanen M, Sipilä P, et al. The accuracy of Acuros XB algorithm for radiation beams traversing a metallic hip implant - comparison with measurements and Monte Carlo calculations. J Appl Clin Med Phys 2014; 15:4912.

4. Failla GA, Wareing T, Archambault Y, Thompson S. Acuros ${ }^{\circledR}$ XB advanced dose calculation for the Eclipse $^{\mathrm{TM}}$ treatment planning system. Clinical perspectives 2010.

5. Wareing TA, McGhee JM, Morel JE, Pautz SD. Discontinuous Finite Element Sn Methods on Three-Dimensional Unstructured Grids. Nucl Sci Eng 2001; 138:256.

6. Wareing TA, Morel JE, McGhee JM. Coupled electron-photon transport methods on 3-D unstructured grids. Trans Am Nucl Soc 2000; 83:240-42.

7. Vassiliev ON, Wareing TA, Davis IM, et al. Feasibility of a multigroup deterministic solution method for three-dimensional radiotherapy dose calculations. Int J Radiat Oncol Biol Phys 2008; 72:220-7.

8. Gifford KA, Horton JL, Wareing TA, Failla G, Mourtada F. Comparison of a finite-element multigroup discrete-ordinates code with Monte Carlo for radiotherapy calculations. Phys Med Biol 2006; 51:2253-65.

9. Vassiliev ON, Wareing TA, McGhee J, Failla G, Salehpour MR, Mourtada F. Validation of a new grid-based Boltzmann equation solver for dose calculation in radiotherapy with photon beams. Phys Med Biol 2010; 55:581-98.

10. Ulmer W, Harder D. A triple Gaussian pencil beam model for photon beam treatment planning. $Z$ Med Phys 1995; 5:25-30.

11. Ulmer W, Harder D. Applications of a triple Gaussian pencil beam model for photon beam treatment planning. Z Med Phys 1996; 6:68-74.

12. Ulmer W, Kaissl W. The inverse problem of a Gaussian convolution and its application to the finite size of the measurement chambers/detectors in photon and proton dosimetry. Phys Med Biol 2003; 48:707-27.

13. Ulmer W, Pyyry J, Kaissl W. A 3D photon superposition/convolution algorithm and its foundation on results of Monte Carlo calculations. Phys Med Biol 2005; 50:1767-90.

14. Tillikainen L, Siljamäki S, Helminen H, et al. Determination of parameters for a multiple-source 
model of megavoltage photon beams using optimization methods. Phys Med Biol 2007; 52:1441-67.

15. Tillikainen L, Helminen H, Torsti T, et al. A 3D pencil-beam-based superposition algorithm for photon dose calculation in heterogeneous media. Phys Med Biol 2008; 53:3821-39.

16. Han T, Mikell JK, Salehpour M, Mourtada F. Dosimetric comparison of Acuros XB deterministic radiation transport method with Monte Carlo and model-based convolution methods in heterogeneous media. Med Phys 2011; 38:2651-64.

17. Bush K, Gagne IM, Zavgorodni S, et al. Dosimetric validation of Acuros XB with Monte Carlo methods for photon dose calculations. Med Phys 2011; 38: 2208-21.

18. Fogliata A, Nicolini G, Clivio A, et al. Dosimetric validation of the Acuros XB Advanced Dose Calculation algorithm: fundamental characterization in water. Phys Med Biol 2011; 56:1879-904.

19. Kan MW, Yu PK, Leung LH. A review on the use of grid-based Boltzmann equation solvers for dose calculation in external photon beam treatment planning. Biomed Res Int 2013; 2013:692874.

20. Chetty IJ, Curran B, Cygler JE, et al. Report of the AAPM Task Group No. 105: Issues associated with clinical implementation of Monte Carlo-based photon and electron external beam treatment planning. Med Phys 2007; 34:4818-53.

21. Hoffmann L, Jørgensen MB, Muren LP, Petersen JB. Clinical validation of the Acuros XB photon dose calculation algorithm, a grid-based Boltzmann equation solver. Acta Oncol 2012; 51:376-85.

22. Mißlbeck M, Kneschaurek P. Comparison between Acuros XB and Brainlab Monte Carlo algorithms for photon dose calculation. Strahlenther Onkol 2012; 188:599-605.

23. Fogliata A, Nicolini G, Clivio A, et al. Accuracy of Acuros XB and AAA dose calculation for small fields with reference to RapidArc(®) stereotactic treatments. Med Phys 2011; 38:6228-37.

24. Torsti T, Korhonen L, Petäjä V. Using Varian Photon Beam Source Model for Dose Calculation of Small Fields. Clinical Perspectives. 2013.

25. Kan MW, Leung LH, Yu PK. The performance of the progressive resolution optimizer (PRO) for RapidArc planning in targets with low-density media. J Appl Clin Med Phys 2013; 14:4382.

26. Kron T, Clivio A, Vanetti E, et al. Small field segments surrounded by large areas only shielded by a multileaf collimator: comparison of experiments and dose calculation. Med Phys 2012; 39:7480-9.

27. Fogliata A, Clivio A, Vanetti E, et al. Dosimetric evaluation of photon dose calculation under jaw and MLC shielding. Med Phys 2013; 40:101706.
28. Tan YI, Metwaly M, Glegg M, Baggarley S, Elliott A. Evaluation of six TPS algorithms in computing entrance and exit doses. J Appl Clin Med Phys 2014; 15:4739.

29. Jeevanandam P, Rajasekaran D, Sukumar P, Nagarajan $\mathrm{V}$. In-house spread sheet based monitor unit verification program for volumetric modulated arc therapy. Phys Med 2014; 30:509-12.

30. Rogers DWO, Mohan R. Questions for comparison of clinical Monte Carlo codes. $13^{\text {th }}$ Int. Conf. on the Use of Computers in Radiotherapy (Heidelberg). 2000:120-2.

31. Kroon PS, Hol S, Essers M. Dosimetric accuracy and clinical quality of Acuros XB and AAA dose calculation algorithm for stereotactic and conventional lung volumetric modulated arc therapy plans. $R a-$ diat Oncol 2013; 8:149.

32. Fogliata A, Nicolini G, Clivio A, et al. Dosimetric evaluation of Acuros XB Advanced Dose Calculation algorithm in heterogeneous media. Radiat Oncol 2011; 6:82.

33. Tsuruta Y, Nakata M, Nakamura M, et al. Dosimetric comparison of Acuros XB, AAA, and XVMC in stereotactic body radiotherapy for lung cancer. Med Phys 2014;41:081715.

34. Stathakis S, Esquivel C, Quino LV, et al. Accuracy of the Small Field Dosimetry Using the Acuros XB Dose Calculation Algorithm within and beyond Heterogeneous Media for $6 \mathrm{MV}$ Photon Beams. Int J Med Phys Clin Eng Radiat Oncol 2012; 1:78-87.

35. Kan MW, Leung LH, Yu PK. Verification and dosimetric impact of Acuros XB algorithm on intensity modulated stereotactic radiotherapy for locally persistent nasopharyngeal carcinoma. Med Phys 2012; 39:4705-14.

36. Kan MW, Leung LH, Yu PK. Dosimetric impact of using the Acuros XB algorithm for intensity modulated radiation therapy and RapidArc planning in nasopharyngeal carcinomas. Int J Radiat Oncol Biol Phys 2013; 85:e73-80.

37. Kan MW, Leung LH, So RW, Yu PK. Experimental verification of the Acuros $\mathrm{XB}$ and AAA dose calculation adjacent to heterogeneous media for IMRT and RapidArc of nasopharygeal carcinoma. Med Phys 2013;40:031714.

38. Rana S, Rogers K. Dosimetric evaluation of Acuros $\mathrm{XB}$ dose calculation algorithm with measurements in predicting doses beyond different air gap thickness for smaller and larger field sizes. JMed Phys 2013; 38:9-14.

39. Rana S, Rogers K, Pokharel S, et al. Acuros XB Algorithm vs. Anisotropic Analytical Algorithm: A Dosimetric Study Using Heterogeneous Phantom and Computed Tomography (CT) Data Sets of Esophageal Cancer Patients. J Cancer Ther 2013; 4:138-44. 
40. Rana S, Rogers K, Lee T, et al. Verification and Dosimetric Impact of Acuros XB Algorithm for Stereotactic Body Radiation Therapy (SBRT) and RapidArc Planning for Non-Small-Cell Lung Cancer (NSCLC) Patients. Int JMed Phys Clin Eng Radiat Oncol 2013; 2:6-14.

41. Rana S, Rogers K, Pokharel S, Cheng C. Evaluation of Acuros XB algorithm based on RTOG 0813 dosimetric criteria for SBRT lung treatment with RapidArc. J Appl Clin Med Phys 2014; 15:4474.

42. Lloyd SA, Ansbacher W. Evaluation of an analytic linear Boltzmann transport equation solver for high-density inhomogeneities. Med Phys 2013; 40:011707.

43. Han T, Mourtada F, Kisling K, et al. Experimental validation of deterministic Acuros XB algorithm for IMRT and VMAT dose calculations with the Radiological Physics Center's head and neck phantom. Med Phys 2012; 39:2193-202.

44. Han T, Followill D, Mikell J, et al. Dosimetric impact of Acuros XB deterministic radiation transport algorithm for heterogeneous dose calculation in lung cancer. Med Phys 2013; 40:051710.

45. Warren S, Panettieri V, Panakis N, et al. Optimizing collimator margins for isotoxically dose-escalated conformal radiation therapy of non-small cell lung cancer. Int J Radiat Oncol Biol Phys 2014; 88: 1148-53.

46. Gimeno J, Pujades MC, García T, et al. Commissioning and initial experience with a commercial software for in vivo volumetric dosimetry. Phys Med 2014: S1120-1797(14)00108-2.

47. Tomiyama Y, Araki F, Kanetake N, et al. Comparison of dose calculation algorithms in stereotactic radiation therapy in lung. Nihon Hoshasen Gijutsu Gakkai Zasshi 2013; 69:663-8.

48. Chetty IJ, Devpura S, Liu D, et al. Correlation of dose computed using different algorithms with local control following stereotactic ablative radiotherapy (SABR)-based treatment of non-small-cell lung cancer. Radiother Oncol 2013; 109:498-504.

49. Fogliata A, Nicolini G, Clivio A, et al. Critical appraisal of Acuros XB and Anisotropic Analytic Algorithm dose calculation in advanced non-small-cell lung cancer treatments. Int J Radiat Oncol Biol Phys 2012; 83:1587-95.

50. Liu HW, Nugent Z, Clayton R, et al. Clinical impact of using the deterministic patient dose calculation algorithm Acuros XB for lung stereotactic body radiation therapy. Acta Oncol 2014; 53:324-9.

51. Khan RF, Villarreal-Barajas E, Lau H, Liu HW. Effect of Acuros XB algorithm on monitor units for stereotactic body radiotherapy planning of lung cancer. Med Dosim 2014; 39:83-7.

52. Fogliata A, Nicolini G, Clivio A, et al. On the dosimetric impact of inhomogeneity management in the Acuros XB algorithm for breast treatment. $R a$ diat Oncol 2011; 6:103.

53. Lonski $\mathrm{P}$, Taylor ML, Hackworth $\mathrm{W}$, et al. In vivo verification of radiation dose delivered to healthy tissue during radiotherapy for breast cancer. J Phys Conf Ser 2014; 489:012015.

54. Petillion S, Swinnen A, Defraene G, et al. The photon dose calculation algorithm used in breast radiotherapy has significant impact on the parameters of radiobiological models. J Appl Clin Med Phys 2014; 15:4853.

55. Rana S, Rogers K, Lee $\mathrm{T}$, et al. Dosimetric impact of Acuros XB dose calculation algorithm in prostate cancer treatment using RapidArc. JCancer Res Ther 2013; 9:430-5.

56. Fogliata A, Scorsetti M, Navarria P, et al. Dosimetric comparison between VMAT with different dose calculation algorithms and protons for soft-tissue sarcoma radiotherapy. Acta Oncol 2013; 52: 545-52.

57. Krayenbuehl J, Riesterer O, Graydon S, et al. Intensity-modulated radiotherapy and volumetric-modulated arc therapy for malignant pleural mesothelioma after extrapleural pleuropneumonectomy. J Appl Clin Med Phys 2013; 14:4130.

58. Kathirvel M, Subramanian S, Clivio A, et al. Critical appraisal of the accuracy of Acuros-XB and Anisotropic Analytical Algorithm compared to measurement and calculations with the compass system in the delivery of RapidArc clinical plans. Radiat Oncol 2013; 8:140.

59. Stroom J, Vieira S, Mateus D, et al. On the robustness of VMAT-SABR treatment plans against isocentre positioning uncertainties. Radiat Oncol 2014; 9:196.

60. Abacioglu U, Ozen Z, Yilmaz M, et al. Critical appraisal of RapidArc radiosurgery with flattening filter free photon beams for benign brain lesions in comparison to GammaKnife: a treatment planning study. Radiat Oncol 2014; 9:119.

61. Fogliata A, Cozzi L, Clivio A, et al. Preclinical assessment of volumetric modulated arc therapy for total marrow irradiation. Int J Radiat Oncol Biol Phys 2011; 80:628-36.

62. Mancosu P, Navarria P, Castagna L, et al. Anatomy driven optimization strategy for total marrow irradiation with a volumetric modulated arc therapy technique. J Appl Clin Med Phys 2012; 13:3653.

63. Essers M, Osman SO, Hol S, et al. Accelerated partial breast irradiation (APBI): are breath-hold and volumetric radiation therapy techniques useful? Acta Oncol 2014; 53:788-94.

64. Osman SO, Hol S, Poortmans PM, Essers M. Volumetric modulated arc therapy and breath-hold in image-guided locoregional left-sided breast irradiation. Radiother Oncol 2014; 112:17-22. 
65. Vanetti E, Nicolini G, Nord J, et al. On the role of the optimization algorithm of RapidArc $\left({ }^{\circledR}\right)$ volumetric modulated arc therapy on plan quality and efficiency. Med Phys 2011; 38:5844-56.

66. Persoon LC, Egelmeer AG, Ollers MC, et al. First clinical results of adaptive radiotherapy based on $3 \mathrm{D}$ portal dosimetry for lung cancer patients with atelectasis treated with volumetric-modulated arc therapy (VMAT). Acta Oncol 2013; 52:1484-9.

67. Gersh JA, Best RC, Watts RJ. The clinical impact of detector choice for beam scanning. J Appl Clin Med Phys 2014; 15:4801. 